\title{
PICTURE QUIZ
}

Journal of the Ceylon College of Physicians, 2012, 43, 61-62

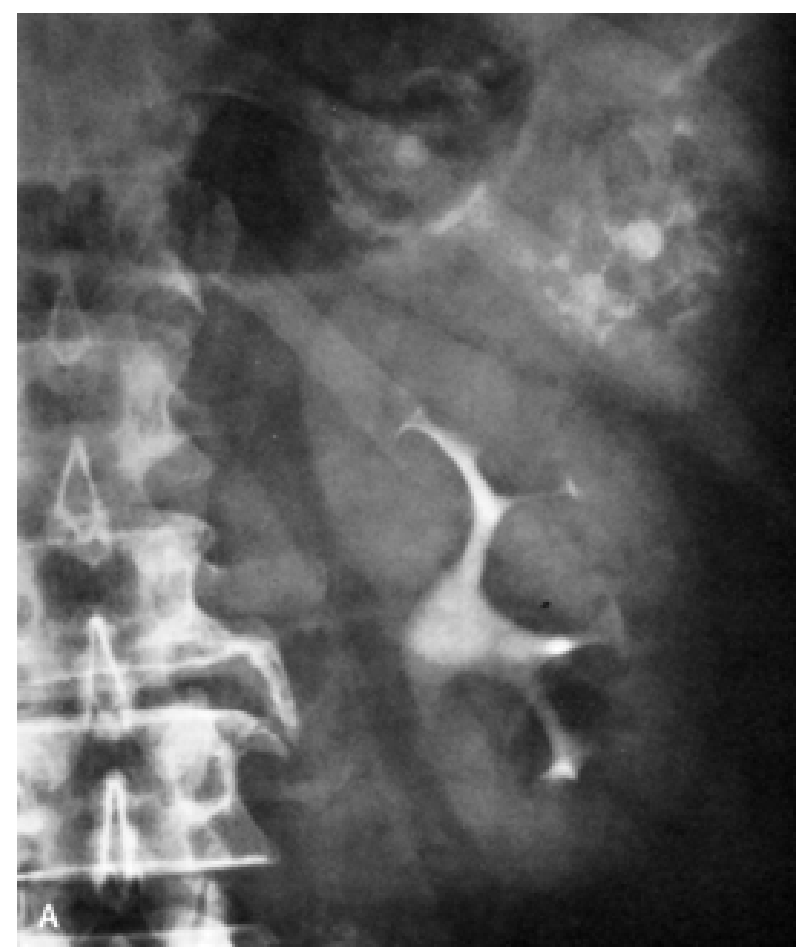

1. A 40-yr-old farmer is investigated for PUO. What is the probable cause?

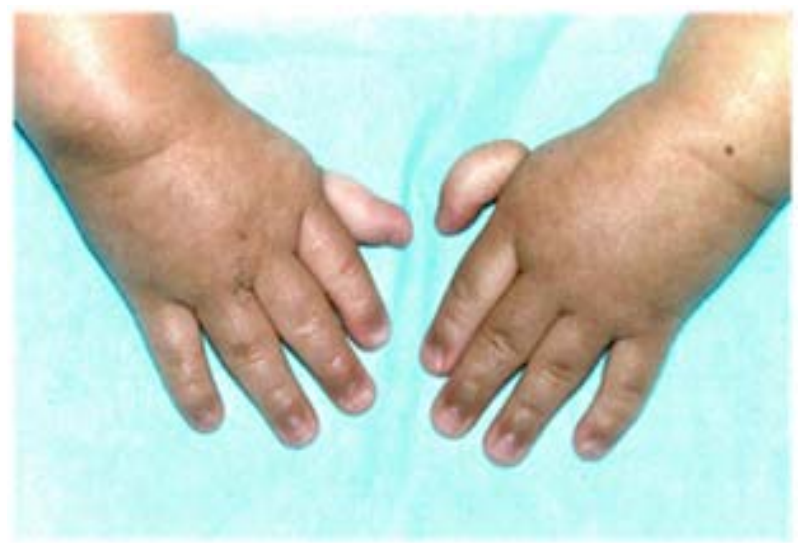

3. This 5-year-old boy was investigated for severe anaemia. What is the underlying diagnosis?

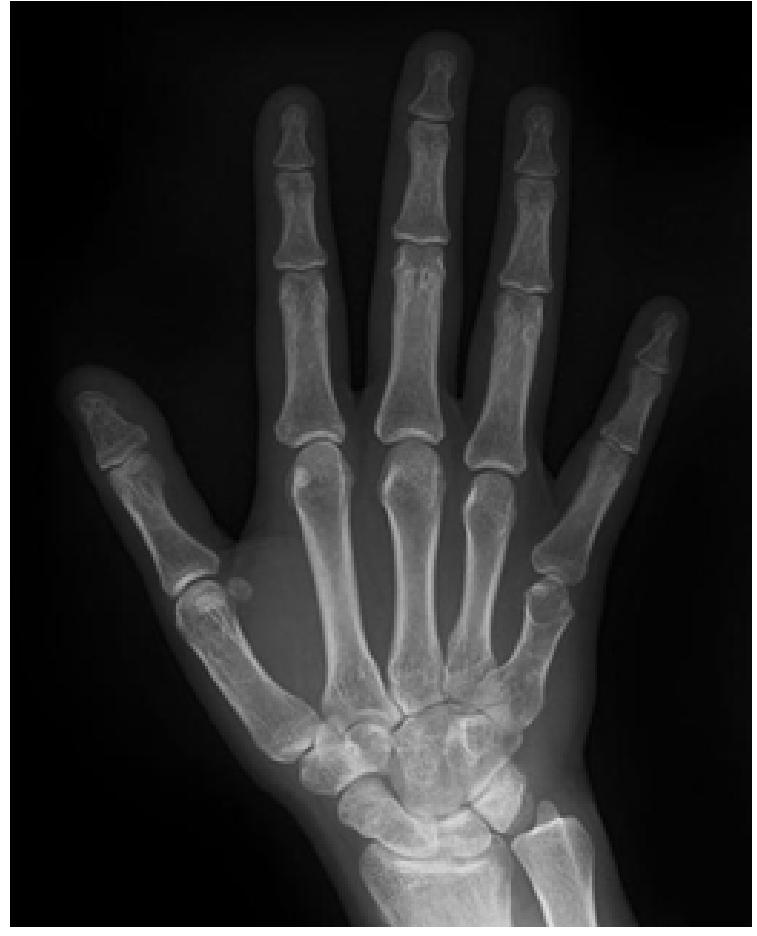

2. What condition is associated with the above Xray abnormality?

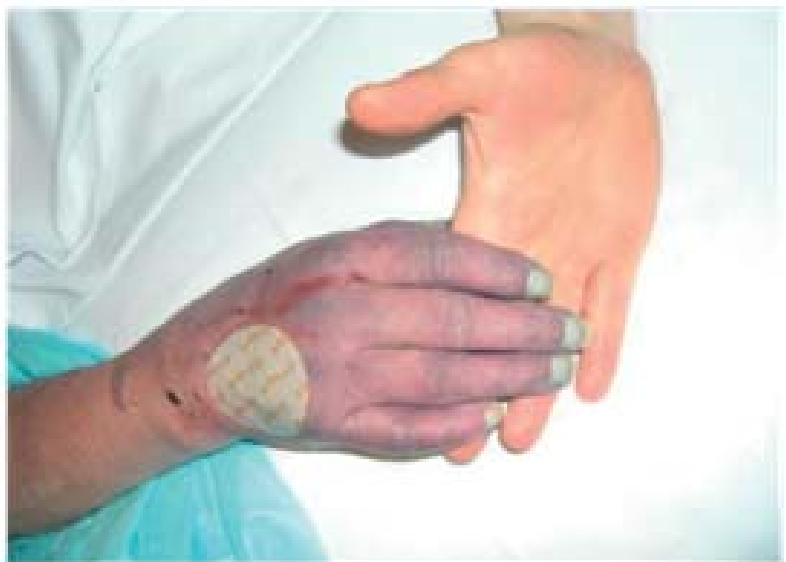

3. This is the hand of a patient who was treated in ICU for Status Epilepticus. What is the diagnosis? 


\section{ANSWERS TO PICTURE QUIZ}

\section{Q1. Brucellosis}

This IVU demonstrates the target ring and spider web pattern calcification in the spleen. Splenic calcifications can occur due to many causes, but the type of lesions differs among causes. Therefore the nature of the calcification is helpful in identifying the underlying cause. "Starry spleen" is a spleen with multiple, punctate calcifications due to tuberculosis, histoplasmosis or brucellosis. Large few calcifications with spider web pattern or target ring lesions are typically seen in brucellosis. These lesions have a calcified rim with a less attenuated center. Tuberculosis produces few fully calcified lesions (typically $<6$ lesions) and histoplasmosis gives rise to larger, numerous (>6) fully calcified lesions with lymph node involvement.

\section{Q2. Insulin resistance Type A}

Isolated short 5th metacarpal bone is uncommon. It has recently been described in a familial variant of Type A diabetes. These patients have a normal 4th metacarpal and do not have the 'metacarpal sign' in hand Xray.

If a line drawn along the heads of the 4th and 5th metacarpal bones intersect the head of the 3rd metacarpal bone, metacarpal sign is said to be present. It is seen in a variety of conditions associated with both short 4th/5th metacarpal bones (Pseudohypoparathyroidism, Turner syndrome, homocystinuria, Pseudopseudohypoparathyroidism) and also in $9.6 \%$ of normal individuals.

\section{Q3. Fanconi anaemia}

This picture demonstrates 'dangling thumb' of Fanconi anaemia. It is a genetic disease inherited as an autosomal recessive trait, originally described by the Swiss Paediatrician, Guido Fanconi. DNA repair is defective resulting in congenital defects (short stature, abnormalities of skin, arms, eyes, kidneys), developmental delay and bone marrow failure. Some patients develop endocrine problems. These patients are at high risk of developing acute myeloid leukaemia and head/ neck or GI malignancy. Definitive treatment is bone marrow transplantation.

\section{Q4. Purple glove syndrome due to Phenytoin}

Purple glove syndrome is a rare but a serious complication of intravenous Phenytoin therapy resulting in painful swelling and discolouration of the extremities. Mechanism is still unknown and three stages of the syndrome is recognized: appearance, progression and resolution. During the second stage, severity can be assessed as mild or severe. Mild cases may heal uneventfully when symptomatic measures are adopted. Severe cases may require emergency surgical intervention such as fasciotomy to relieve pressure and restore blood flow. Early identification could minimize the complications.

Compiled by Nilanka Perera, Lecturer in Medicine, USJP 